\title{
Hemoperitoneo secundario a hemocolecisto por colecistitis aguda
}

\author{
Hemoperitoneum secondary to haemocholecyst due to acute cholecystitis \\ Elena Sagarra-Cebolla*, Sergio P. Olivares-Pizarro, Raquel Ríos-Blanco, \\ Eduardo de San-Pío-Carvajal e Ignacio Rodríguez-Prieto \\ Servicio de Cirugía General y del Aparato Digestivo, Hospital Universitario Infanta Cristina, Parla, Madrid, España
}

\section{Resumen}

El hemocolecisto es una patología poco frecuente y con un índice bajo de sospecha, que todavía es menor si el paciente ho presenta factores de riesgo. Asimismo, su curso con clínica de hemoperitoneo sin perforación vesicular es igualmente infrecuente. Presentamos un caso poco habitual en el cual el diagnóstico de la paciente se llevó a cabo intraoperatoriamënte. Consideramos que comunicar estos casos poco habituales en la práctica clínica colabora a aumentar la sospecha clínica y el diagnóstico precoz.

Palabras clave: Colecistitis aguda. Hemocolecisto. Hemoperitoneo.

\section{Abstract}

The haemocholecyst is a rare disease with a low index of suspiction, which is even lower if the patient does not presenterisk factors. Likewise, the course of this pathology with a haemoperitoneum without vesicular perforation is equally infrequent? We present an unusual case in which the diagnosis of the patient was carried out intraoperatively. We consider that communicating these unusual cases in clinical practice helps to increase clinical suspiction and prompt diagnosis.

Key words: Acute cholecystitis. Haemocholecyst. Haemoperitoneum.

\section{Introducción}

El hemocolecisto es un término introducido en 1961 por Fitzpatrick ${ }^{1,2}$, el cual define la hemorragia de la vesícula biliar. Es una patología poco frecuente ${ }^{3}$ que puede cursar con una gran variabilidad clínica, siendo difícil diferenciarla de la hemobilia ${ }^{3}$.

\section{Caso clínico}

Mujer de 54 años, con antecedentes personales de hipertensión arterial, diabetes mellitus, dislipidemia, diabetes insípida central e insuficiencia suprarrenal secundaria a resección de microadenoma hipofisariofuncionante. La paciente acudió al servicio de urgenéias 
de nuestro centro por dolor epigástrico de 3 días de evolución asociado a febrícula sin otra clínica acompañante. En la analítica de sangre destacaba leucocitosis con elevación de los reactantes de fase aguda, sin alteración del perfil hepático, con una hemoglobina de 13.5. Se realizó ecografía abdominal que evidenció una marcada distensión de la vesícula biliar, cuya luz estaba ocupada completamente por material hiperecogénico y una imagen con sombra acústica posterior compatible con colelitiasis. El espesor máximo de la pared era de 3-4 mm. El Murphy ecográfico era negativo. Se objetivó una mínima lengüeta de líquido perivesicular adyacente al fundus. La paciente inició tratamiento antibiótico empírico con amoxicilina-ácido clavulánico y presentó una buena evolución analítica en las primeras horas; sin embargo, ante el empeoramiento y la generalización del dolor abdominal con irritación peritoneal, se decidió tratamiento quirúrgico urgente a las 28 horas de su ingreso en el servicio de urgencias. Se realizó abordaje laparoscópico. El hallazgo intraoperatorio fue un hemoperitoneo de los cuatro cuadrantes, de unos $500 \mathrm{ml}$ hemáticos. La vesícula presentaba engrosamiento de la pared y un voluminoso cálculo en su interior, sin perforación (Fig. 1). Al vaciarla percutáneamente para facilitar su manipulación se obtuvo hemobilia. La colecistectomía se completó laparoscópicamente sin incidencias.

La evolución posoperatoria fue favorable y se mantuvo la antibioticoterapia empírica intravenosa durante el ingreso. Fue dada de alta al tercer día de posoperatorio sin evidenciar anemización, manteniendo la cifra de hemoglobina en 13.1, y sin otras alteraciones analíticas. Completó el ciclo de 5 días de amoxicilina-ácido clavulánico.

En el informe anatomopatológico, el interior de la vesícula presentaba una litiasis y abundante material hemático que ocupaba completamente la luz, evidenciando en el estudio microscópico la ausencia de revestimiento epitelial de la mucosa.

\section{Discusión}

El hemocolecisto es una patología que se ha asociado con distintas etiologías, pudiendo ser secundaria a colecistitis, neoplasia de la vesícula biliar, aneurisma de la arteria cística, postraumática, isquémica, infecciosa por parasitosis y por alteraciones de la coagulación, incluyendo aquellas discrasias debidas a enfermedad renal, cirrosis hepática o por tratamientos anticoagulantes y antiagregantes ${ }^{2-4}$. El sangrado de la vesícula biliar por colecistitis es poco habitual debido

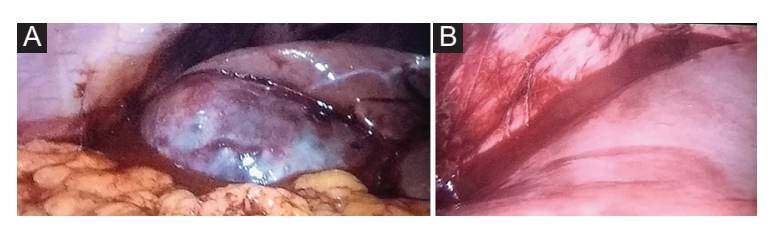

Figura 1. A: vesícula biliar a tensión con engrosamiento de la pared secundaria a la colecistitis y hemoperitoneo en el espacio de Moprison. B: hemoperitoneo en el espacio subdiafragmático derecho.

a que la inflamación causa, principalmente, trombösis vascular. Sin embargo, también puede causar daño a la adventicia de los vasos, debilitando su pared ỹgenerando el sangrado ${ }^{4,5}$. Asimismo, el sangrado secundario a la colecistitis puede ser producido por ulceración y necrosis de la mucosa o por afectación del leêno hepático al producirse la perforación de la vesícu敢

Se han descrito distintas presentaciones del hemocolecisto $^{3}$. El sangrado puede producir coágulos intravesiculares que condicionan distensión vesicular y una clínica típica de colecistitis aguda ${ }^{1,2,4,5}$. Si esta evoluciona, se puede producir necrosis de la pared con perforación vesicular, dando origen a un abdomen agudo secundario al hemoperitoneo $0^{1,2,5}$. El hemoperitóneo también puede ser secundario a la erosión de la artèria cística o de una de sus ramas ${ }^{4,5}$. La sangre intravesicular también puede progresar hacia la vía biliâr y obstruirla, favoreciendo el desarrollo de colangitis o, si llega hasta el tracto digestivo, dando clínica de hemnrragia digestiva alta ${ }^{1,2,3,5}$. En la tabla 1 se comparan nuestros hallazgos con los descritos en la literatuià.

El diagnóstico es difícil y generalmente se reajiza de manera intraoperatoria' ${ }^{1}$. Los hallazgos ecográficos incluyen un engrosamiento o irregularidades focales de la pared de la vesícula biliar y la existencià de material ecogénico intraluminal sin sombra acústica posterior $^{2-5}$. En la tomografía computarizada se observa líquido de alta densidad en la luz de la vesículla, pudiendo demostrarse la extravasación del contrăste en la fase arterial precoz en caso de sangrado activo significativo. Esta extravasación puede pasar dẹsapercibida en la fase tardía ${ }^{2,3,5}$. Cuando existe hemoperitoneo asociado se evidencia líquido hiperdeñso alrededor de la vesícula, que puede estar acompañado de un defecto en la pared de esta e incluso de salida de litiasis a la cavidad abdominal si hay peirforación de la pared vesicular ${ }^{5}$. La colangiopancreâtografía retrógrada endoscópica puede evidenciạ el sangrado a través de la ampolla duodenal, aunque este solo aparece en el $30 \%$ de los casos $^{3}$. 
Tabla 1. Cuadro comparativo de nuestra paciente y los casos clínicos revisados

\begin{tabular}{|c|c|c|c|c|}
\hline Factores de riesgo & Motivo de consulta & Pruebas complementarias & Abordaje & ชั่ \\
\hline $\begin{array}{l}\text { Sin factores de riesgo para } \\
\text { sangrado ni enfermedad } \\
\text { hepática conocida }\end{array}$ & Dolor en hipocondrio derecho & $\begin{array}{l}\text { Analíticos: hemoglobina 13.5, leucocitosis } \\
\text { Ecografía: colecistitis con ocupación de la luz } \\
\text { vesicular }\end{array}$ & Laparoscópico & 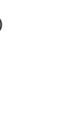 \\
\hline Clopidogrel y acenocumarol & Dolor en hipocondrio derecho & $\begin{array}{l}\text { Analíticos: hemoglobina 10.8, leucocitosis, } \\
\text { alteración perfil hepatobiliar } \\
\text { Ecografía: distensión de la vesícula con } \\
\text { ocupación }\end{array}$ & Abierto & 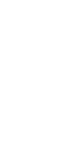 \\
\hline $\begin{array}{l}\text { Cirrosis hepática de origen } \\
\text { enólico, acenocumarol }\end{array}$ & Hemorragia digestiva alta & $\begin{array}{l}\text { Analíticos: hemoglobina } 9.8 \text {, leucocitosis, } \\
\text { alteración perfil hepatobiliar } \\
\text { Tomografía: distensión de la vesícula con } \\
\text { ocupación }\end{array}$ & Laparoscópico & 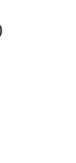 \\
\hline $\begin{array}{l}\text { Ácido acetilsalicílico, heparina } \\
\text { de bajo peso molecular a dosis } \\
\text { profiláctica }\end{array}$ & Hemorragia digestiva baja & $\begin{array}{l}\text { Analíticos: hemoglobina 8, leucocitosis, } \\
\text { alteración perfil hepatobiliar } \\
\text { Tomografía: colecistitis con material de } \\
\text { densidad sangre en el interior de la vesícula } \\
\text { y dilatación de la vía biliar }\end{array}$ & $\begin{array}{l}\text { Laparoscópico } \\
\text { reconvertido a a }\end{array}$ & $\begin{array}{l}\frac{0}{\circ} \\
\text { abierto } \\
\underset{\frac{\pi}{\pi}}{\frac{\pi}{\pi}} \\
\frac{2}{\sigma} \\
\frac{\sigma}{5} \\
\frac{\sigma}{5}\end{array}$ \\
\hline $\begin{array}{l}\text { Cirrosis hepática secundaria a } \\
\text { cirrosis esclerosante primaria en } \\
\text { paciente con colitis ulcerosa }\end{array}$ & Dolor en hipocondrio derecho & $\begin{array}{l}\text { Analíticos: hemoglobina 13.6, leucocitosis, } \\
\text { alteración perfil hepatobiliar } \\
\text { Tomografía: hematoma en el lecho vesicular con } \\
\text { compresión de la vesícula y sangrado activo }\end{array}$ & Abierto & 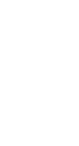 \\
\hline
\end{tabular}

El tratamiento de esta patología depende de su etiología ${ }^{3}$. Cuando el origen es traumático o secundario a una discrasia sanguínea se puede tratar de manera conservadora siempre que el paciente se encuentre hemodinámicamente estable, presente mejoría clínica y no se evidencien signos de sangrado activo $^{3}$. La colecistectomía urgente está indicada en el caso de que la etiología sea una colecistitis aguda ${ }^{3}$ o si existe inestabilidad hemodinámica ${ }^{1,2}$. Si se sospecha una neoplasia está indicada la colecistectomía electiva ${ }^{3}$. La colecistostomía percutánea es una alternativa válida en pacientes no candidatos a tratamiento quirúrgico para evitar la perforación de la vesícula secundaria a su distensión por la sangre ${ }^{2}$. Las complicaciones posoperatorias más habituales son la hemorragia y el shock hipovolémico².

\section{Agradecimientos}

Los autores agradecen al personal de los servicios de urgencias y quirófano de guardia del Hospital General Universitario Infanta Cristina de Parla.

\section{Responsabilidades éticas}

Protección de personas y animales. Los autores declaran que los procedimientos seguidos se conformaron a las normas éticas del comité de experimentación humana responsable y de acuèrdo con la Asociación Médica Mundial y la Declaración de Helsinki.

Confidencialidad de los datos. Los autores declaran que han seguido los protocolos de su centro de trabajo sobre la publicación de datos de pacientes.

Derecho a la privacidad y consentimiento informado. Los autores han obtenido el consentimiënto informado de los pacientes y/o sujetos referidos en el artículo. Este documento obra en poder del autor de correspondencia.

\section{Conflicto de intereses}

Los autores declaran que no existe ningún conflicto de intereses en la realización de este trabajo.

\section{Bibliografía}

1. Polse S, Stoney RJ, Baldwin JN. Hemorrhage from the gallbladder. A report of three cases. Calif Med. 1967;107:51-3.

2. Ángel JM, Bermúdez RP, Conde MA, Sampedro JE, Jofré JG. Haemo-cholecyst as a complication of anticoagulant and anti-platelet treatment. Cir Esp. 2011;89:327-9.

3. Barbon Remis E, Martínez Menéndez A, Alexandre Hurle E, Del Casar Lizcano JM, Díez Santesteban MC. Laparoscopic treatment of a hemocholecyst. Cir Esp. 2014;92:293-5.

4. Hicks N. Haemorrhagic cholecystitis: an unusual cause of upper gastrointestinal bleeding. BMJ Case Rep. 2014;2014:bcr2013202437.

5. Aljiffry MM, Almulhim AN, Jamal MH, Hassanain MM. Acute cholecystitis presenting with massive intra-abdominal haemorrhage. J Surg Case Rep. 2014;2014:rju019. 